\title{
Discovery of old diseases: the molecular approach
}

\author{
European Journal of Human Genetics (2003) 11, 3-4. doi:10.1038/sj.ejhg.5200917
}

It has been a real surprise for us to discover diseases in Cyprus that have remained neglected, not through lack of clinical significance, but mainly through ignorance among most practicing clinicians. A situation that seems to derive from two main reasons. One is that most Cypriot doctors attend medical schools/universities and train for their specialisation in countries of central or northern Europe and northern America, where these diseases are indeed rare. The second is perhaps related more to the unavailability of technology and laboratory facilities for assisting diagnosis, especially in cases where clinical diagnosis is not always straightforward.

The most striking case at hand recently, is Familial Mediterranean Fever (FMF), an inherited inflammatory condition, not lethal in most instances nowadays, but quite painful and debilitating, usually when an incorrect diagnosis is made and treatment instituted. In ethnic groups where FMF is historically considered to be very common (Jews, Arabs, Turks and Armenians) and perhaps with more easily recognisable clinical presentation, and where doctors, for various reasons are more sensitised and inclined to include FMF in their differential diagnosis, the disease has been studied for decades at the clinical level, and more recently at molecular level. The presence of universities and researchers always help the few scientists who are research-oriented to investigate further any suspicions they may have on scientific or medical matters and make proper announcements informing and educating the rest of the medical community.

In Cyprus, there had been no University until 1992, and there is still no medical school, whereas a school of biological sciences has just been created. Therefore, no proper forum for real medical or clinical research has ever existed, although there have always been those few who aspired by an uneasy mind, which was cultivated during their training elsewhere, made efforts to investigate matters of clinical importance among their busy clinical practice. Until three or four years ago, there were less than 30 clinically diagnosed Greek-Cypriot FMF patients, and most of them were diagnosed out of the clinical practice of only few rheumatologists who also considered FMF a rather rare condition on the island. In 1962 Dr Partellides reported on two cases, one a Greek-Cypriot woman and the other a Turkish-Cypriot woman, who presented with periodic fever. In his concluding remarks he commented that FMF was very rare in Cyprus, when it was very common in the Near East, also known as 'Armenian Disease'. ${ }^{1}$

Molecular investigation proved $M E F V$ gene mutations to be frequent among the general Cypriot population. The frequency of three mutations, V726A, M694V and F479L (a rather Cypriot mutation) is $1: 25$, whereas if one includes the milder mutation $\mathrm{E} 148 \mathrm{Q}$, the frequency climbs to the striking 1:9 (Ref. 2 and unpublished data). The clinical contribution of mutation E148Q is under investigation, however it is already known that in homozygosity it only leads to a diagnosis of phenotype half of the time, perhaps because of rare episodes, whereas in heterozygosity with another mutation, it most often leads to typical or atypical recurrent episodes of FMF. Since we introduced molecular testing for $M E F V$ gene mutations two years ago and we informed doctors of the probable high frequency of FMF in Cyprus, more and more samples started reaching our laboratory, requiring investigation. In fact, among a total of over 60 patients presently in our own registry, more than half were diagnosed during the past 2 years, after the combination of clinical suspicion and molecular testing. This observation strengthens the hypothesis, not popular among many clinicians, that FMF diagnosis is not strictly clinical, on the contrary, molecular documentation is a great help in many cases. This, of course, may be associated also with the combination of specific $M E F V$ genetic defects and the general genetic background, as it is known that other genes can modify the clinical picture and the spectrum of symptoms.

With one out of nine Greek-Cypriots being a carrier of an $M E F V$ mutation, we anticipate that many hundreds of patients exist undiagnosed and suffering recurrent episodes of fever, strong incapacitating abdominal pains and unnecessary operations. Cyprus is a small island with a population of about 750000 , and we are in a strong position to suspect that only a very small number of patients have been clinically diagnosed and received medication, however most, have not yet come to our attention. Let us also add here, that testing of anonymous samples in our DNA database identified some that carry two MEFV mutations, hence one expects that these people should have a clinical phenotype, yet, to our knowledge, remain undiagnosed. I wish to emphasize therefore, that conditions like FMF that are not easily diagnosed by expert medical doctors simply because they do not include it in their differential 
diagnosis, can go unnoticed for decades and many patients are misdiagnosed and mistreated, until the word is spread through general awareness programmes. When that happens, suddenly many patients reach the laboratory on their own asking for testing or many doctors will start referring old and new patients who partly or fully fulfill the criteria, and FMF enters their exercise of differential diagnosis. Epidemiological studies, therefore, aimed at identifying disease causing mutations and their general population frequency, assist in evaluating the degree of alertness specialists should have (paediatricians, gastrenterologists, rheumatologists) in exercising medical practice and interpreting symptoms. Apparently, in the classic populations with high prevalence of FMF, almost every clinician is adequately sensitized and bears in mind that a certain spectrum of symptoms is most probably explained by FMF, thereby asking for additional helpful tests aimed at establishing or excluding the diagnosis, whereas the introduction of molecular testing during the last four years has been instrumental in resolving especially atypical cases. We cannot emphasize enough that correct early diagnosis is of paramount importance, both for sparing the patient unnecessary operations, and for eliminating the suffering and increased risk for amyloidosis that can lead to serious kidney failure.
Let us end this note with the conclusion that $M E F V$ mutations and FMF must have been frequent in Cyprus for many hundreds if not thousands of years, but nevertheless, it was only very recently that this was scientifically documented and recognised. The discovery of the high frequency of this apparently very old disease was made possible through the use of molecular diagnostics, which once again proved to be a very powerful tool. It would be interesting to know what the experience has been in other countries and populations of the so-called non-classic ancestry.

C Constantinou Deltas

Department of Biological Sciences, University of Cyprus, and Department of Molecular Genetics, The Cyprus Institute of Neurology and Genetics, Nicosia, Cyprus

E-mail: Deltas@ucy.ac.cy

\section{References}

1 Partellides G: Two cases of periodic disease in Cyprus. Iatrikon Periodikon Kyprou 1962; XII (3): 77-81.

2 Constantinou Deltas C et al: Familial Mediterranean fever (FMF) is a frequent disease in the Greek-Cypriot population of Cyprus. Genetic Testing 2002; 6 (1): 15-21. 